\title{
Effect of Concave Wall Geometry on Heat Transfer in Hypersonic Boundary Layers
}

\author{
W. Flaherty* and J.M. Austin ${ }^{\dagger}$ \\ Department of Aerospace Engineering, University of Illinois at Urbana-Champaign, 61801
}

\begin{abstract}
Heat transfer measurements are made to investigate the effects of concave surface curvature on a high-stagnation enthalpy boundary layer in a Mach 5.1 flow. Experiments are carried out using two curved models with 16 and 25 degree turning angles, and baseline planar models (flat plate and linear ramp) for comparative study. Streamwise and spanwise cross-sections are obtained. Significant destabilization of the boundary layer is observed over the adverse pressure gradient geometries. For the curved surfaces, the heat flux distribution appears to exhibit a quadratic dependence with streamwise distance, in contrast with the linear dependence observed on the linear ramp.
\end{abstract}

\section{Introduction}

As a boundary layer develops over a surface with concave wall curvature, it is subject to compound destabilizing mechanisms such as an adverse pressure gradient, streamline curvature and compression, and possibly centrifugal instabilities such as Görtler vortices. This study is part of an ongoing work experimentally investigating the response of a high-enthalpy, hypersonic boundary layer to concave wall curvature. In this paper, we focus on heat transfer measurements in laminar boundary layers.

In hypersonic flight, concave wall geometries are of current interest due to developments in the design of inward turning inlets. ${ }^{1-4}$ Inward turning inlet designs have been chosen for both the HyCause series, ${ }^{5}$ and the Falcon engine. ${ }^{6}$ These designs assume isentropic compression such that ideally, when operating at the design condition, the only stagnation pressure loss in the system is across the conical shock created at the point where the compression waves coalesce. ${ }^{7}$ This serves to increase the efficiency of the inlet over typical planar configurations. ${ }^{8,9}$ An elliptical cross section is also desirable for the combustor due to increased structural integrity and reduced skin friction. While the theoretical efficiency of the vehicle is increased using inward turning inlets, the flowfield is complicated by the introduction of wall curvature which may significantly impact the boundary layer, a flow known to be susceptible to three-dimensional effects.

Donovan et al. ${ }^{10}$ investigated the response of a supersonic (Mach 2.86), turbulent boundary layer to a segment of curved surface. The combination of the concave geometry, adverse pressure gradient, and bulk compression was found to increase the Reynolds stresses over similar flat plate models which were subjected to adverse pressure gradients only. The adverse pressure gradient created from wall curvature was observed to cause a $125 \%$ increase in wall shear stress, a result which is opposite that in a subsonic flow. ${ }^{10}$ Significant effects on the turbulent structures were also detected in the presence of concave wall curvature; structure angles increased and the streamwise extent of the average large-scale motion nearly doubled.

The response of mean and turbulent flow structures in a supersonic (Mach 2.87) boundary layer to local (surface roughness) and global (streamwise curvature) mechanical distortion was investigated by Ekoto et al. ${ }^{11}$ Several wall geometries were investigated, including a combined pressure gradient model consisting of an expansion section followed by a recompression. For this wall geometry, the sign of all extra strain rates was reversed in comparison with a favorable pressure gradient model. The distortion was weaker near the wall than in the outer region.

Holden and Chadwick ${ }^{12}$ studied boundary layers which were observed to span laminar to turbulent conditions over a concave, curved surface. Wall pressure and heat transfer were measured in Mach 10, 11

\footnotetext{
*Graduate Student, Department of Aerospace Engineering, University of Illinois at Urbana-Champaign

$\dagger$ Assistant Professor, Department of Aerospace Engineering, University of Illinois at Urbana-Champaign
} 
and 12 freestreams. There are two results from this study which are particularly important to the present work. First, the heat transfer was found to increase along a curved ramp. Second, good agreement was obtained with numerical simulations as long as the point of transition was well modeled.

Ciolkosz and Spina ${ }^{13}$ used micro-carbon particulate visualization to investigate the existence of vortex structures in supersonic flow. They were able to determine the existence of longitudinally-oriented vortices, which were taken to be Görtler vortices. They also found that the vortices exist only above a critical Görtler number, and that this critical value increases with increasing Mach number. de Luca et al. ${ }^{14}$ investigated the effects of Görtler vortices on wall heat transfer at Mach 7. They detected spanwise periodic increases in heat transfer which were associated with the formation of Görtler vortices along the surface. It was found that these fluctuations caused variations in the heat flux between $20-30 \%$.

In the present work, we experimentally investigate laminar boundary layers in high-stagnation enthalpy, hypersonic flow. We carry out a comparative study using two models with different Görtler number, and two baseline models: a flat plate and a linear ramp. The response of the boundary layer heat flux to wall geometry is quantified.

\section{Experimental Setup}

The HET facility is an $9.14 \mathrm{~m}$ long expansion tube consisting of driver, driven, and accelerator sections all with a $150 \mathrm{~mm}$ inner diameter. ${ }^{15}$ The facility accesses a range of test conditions with stagnation enthalpies from 4 to $8 \mathrm{MJ} / \mathrm{kg}$ and Mach numbers from 3.0 to 7.5. For this study, a single test condition was used for all the models. Inviscid, perfect gas dynamic calculations are used to predict test gas conditions, shown in Table 1.

Table 1: Theoretical parameters for HET run conditions.

\begin{tabular}{cc}
\hline \hline Condition & Air 4 \\
\hline Mach number & 5.12 \\
Static temperature, $\mathrm{K}$ & 676 \\
Static pressure, $\mathrm{kPa}$ & 8.13 \\
Velocity, $\mathrm{m} / \mathrm{s}$ & 2664 \\
Density, $\mathrm{kg} / \mathrm{m}^{3}$ & 0.042 \\
Test time, $\mu \mathrm{s}$ & 361 \\
Unit Reynolds number, $1 / \mathrm{m}$ & $3.42 \mathrm{e} 6$ \\
Stagnation enthalpy, $\mathrm{MJ} / \mathrm{kg}$ & 4.08 \\
Initial Pressures, $\mathrm{kPa}$ & \\
${ } }$ & 2500 \\
Driven section & 6.0 \\
Accelerator section & 0.08
\end{tabular}

The heat transfer gages used in these experiments are thermocouples based on the design of Sanderson. ${ }^{16}$ They are coaxial, $2.4 \mathrm{~mm}$ in diameter, type E (Constantan-Chromel), and mount flush with the surface of a model. The electrodes can be seen disassembled in Figure 1. The two coaxial elements are designed such that an extremely thin junction (on the order of $1 \mu \mathrm{m}$ ) is formed at the surface. This type of thermocouple gauge is used extensively in the T5 reflected shock tunnel at GALCIT. ${ }^{16-18}$ The high enthalpy test conditions result in adequate signal levels and the robust design of the gages make them highly resistant to damage caused by particulates in the test gas as well as the large heat fluxes. ${ }^{16}$ The output signal is processed by a differential amplifier circuit mounted exterior to the test section. The circuit gain is 1000 to maximize signal amplitude. Individual calibration of thermocouples is not necessary, since the temperature response of all common thermocouple types is well known. The NIST thermocouple reference tables were used to convert from voltage to temperature. ${ }^{19}$ The method of heat transfer deconvolution is described in Flaherty and Austin, ${ }^{20}$ and is based on the work of Sanderson. ${ }^{16}$ 


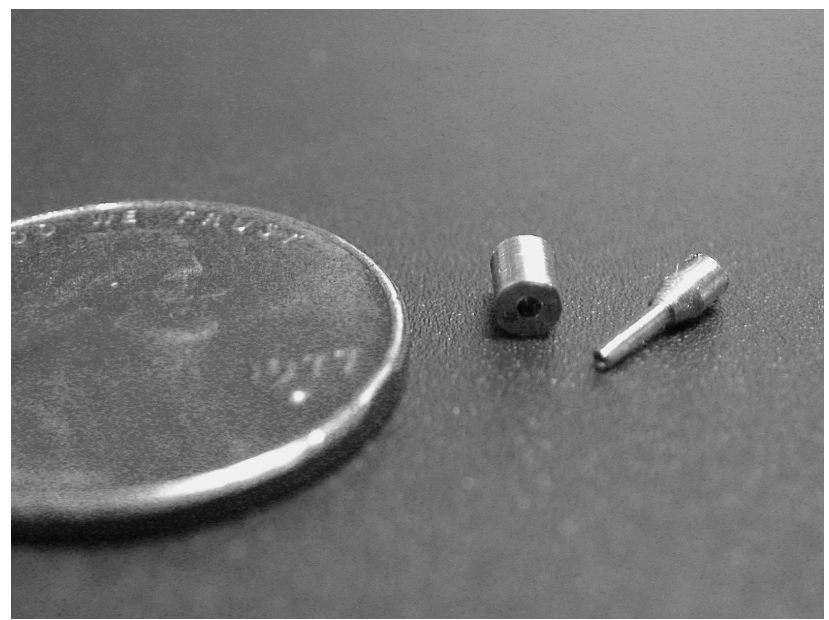

Figure 1: Coaxial thermocouples were used for heat flux measurements.

Four different models were used in for these experiment: a flat plate, a linear ramp, and two curved ramp models. Models were designed to be interchangeable such that the same leading edge was used in all experiments. Each was instrumented with both thermocouple and pressure transducer arrays. The flat plate, shown in Figure 2a, was chosen as a baseline case since data could be compared with existing theoretical models.

The first curved ramp model (denoted Curved25), shown in Figure 2b, was designed with two conditions in mind. There is a flat plate section after the leading edge, and curvature does not begin until $83 \mathrm{~mm}$ down the plate. This was done to allow the boundary layer to form over the model before the curved section was encounted. The radius of curvature was selected using theoretical calculations of Görtler number( based on the method described by Luca et al. ${ }^{14}$ ). Using this method, the Görtler number over Curved25 was calculated to vary between 12 and 15. This magnitude was chosen based on the results of Ciolkosz and Spina $^{13}$ who showed that in a compressible flow Görtler vortices were observed between Görtler numbers of 6 and 13. Since increasing Mach number should have a stabilizing effect on the flow it was decided that the curved ramps should have Görtler numbers on the high end of this range. The overall turning angle of this model is 25 degrees.

The second curved ramp model (Curved16), seen in Figure 3a, was designed to recreate the ramp used by Donovan to investigate the effects of wall curvature in a Mach 2.86 flow. ${ }^{10}$ Again, the model was designed with an initial flat section in order to match the Reynolds number at the beginning of curvature for each model. The flat section is followed by a curved segment with a turning angle of 16 degrees, followed by a final, linear section. The linear ramp (Linear10), seen in Figure 3b, was built as a second baseline model. Comparisons of linear and curved ramp data will be used to assess the effects of wall curvature. The turning angle of the ramp was set at 10.5 degrees by requiring that the hight of the linear ramp be the same as the hight of the first curved ramp model. The characteristics of each model are listed in Table 2

\begin{tabular}{|c|c|c|c|c|}
\hline & Flat Plate & Curved25 & Curved16 & Linear10 \\
\hline Length of model $(\mathrm{m})$ & 0.248 & 0.248 & 0.248 & 0.248 \\
\hline Length of curvature $(\mathrm{m})$ & - & 0.14 & 0.1 & - \\
\hline Turning angle (degrees) & - & 25 & 16 & 10.5 \\
\hline Radius of curvature $(\mathrm{m})$ & - & 0.33 & 0.35 & - \\
\hline \# of thermocouples & 8 & 5 & 14 & 9 \\
\hline \# of pressure transducers & 0 & 0 & 4 & 4 \\
\hline
\end{tabular}

Table 2: Model parameters 


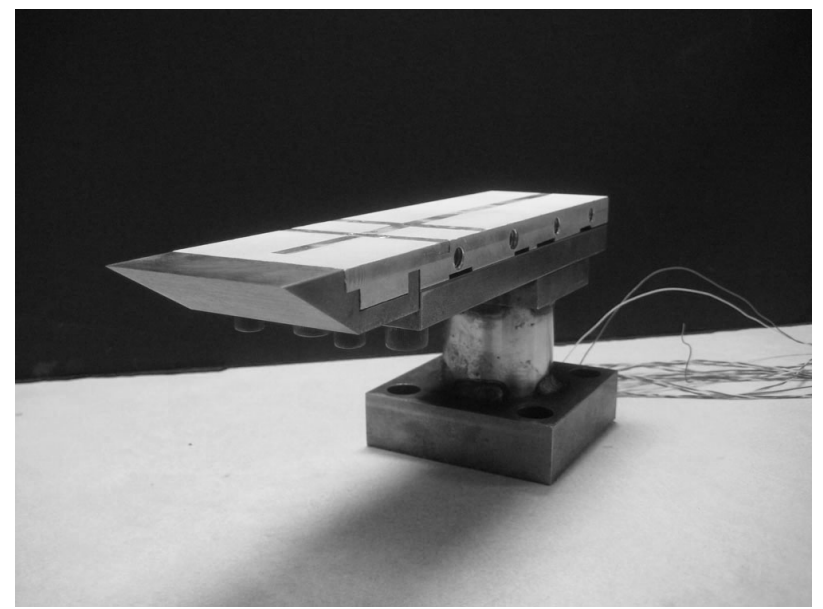

(a)



(b)

Figure 2: (a) Flat plate and (b)curved ramp (Curved25) models.

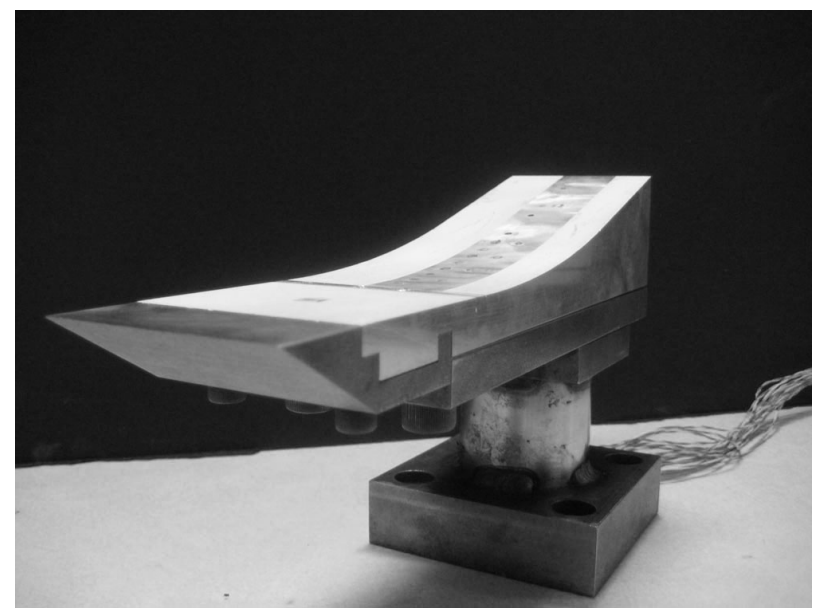

(a) Curved16.



(b) Linear10

Figure 3: (a) Curved ramp (Curved16) and (b) linear ramp models.

\section{Error Analysis}

Davis $^{17}$ calculated the sources of uncertainty for the thermocouple gages designed by Sanderson. Two main sources of uncertainty were identified. First, there is error in the voltage-to-temperature conversion due to uncertainty in the NIST temperature conversion tables. Davis reports this to be $1.7 \%$ in the temperature change, which corresponds directly to a $1.7 \%$ error in the heat flux. Secondly, there is uncertainty in the thermal properties of the thermocouple materials. Davis was able to determine that the uncertainty of the thermal properties (as applied to the calculation of heat flux) was $8 \%$. These values were used directly in this work since both the design and material choice were the same as used by Davis.

It is important to note that this error analysis takes into account the physical uncertainties associated with the gage alone. There is also shot-to-shot variability in the test conditions. Small variations in initial pressures in the facility can cause fluctuations in the free stream properties ${ }^{21}$ and therefore in the heat transfer. In addition, the free stream conditions have some unsteadiness during the test time. Heat transfer results presented here are averaged over the test time, which was experimentally measured using pitot probes. 


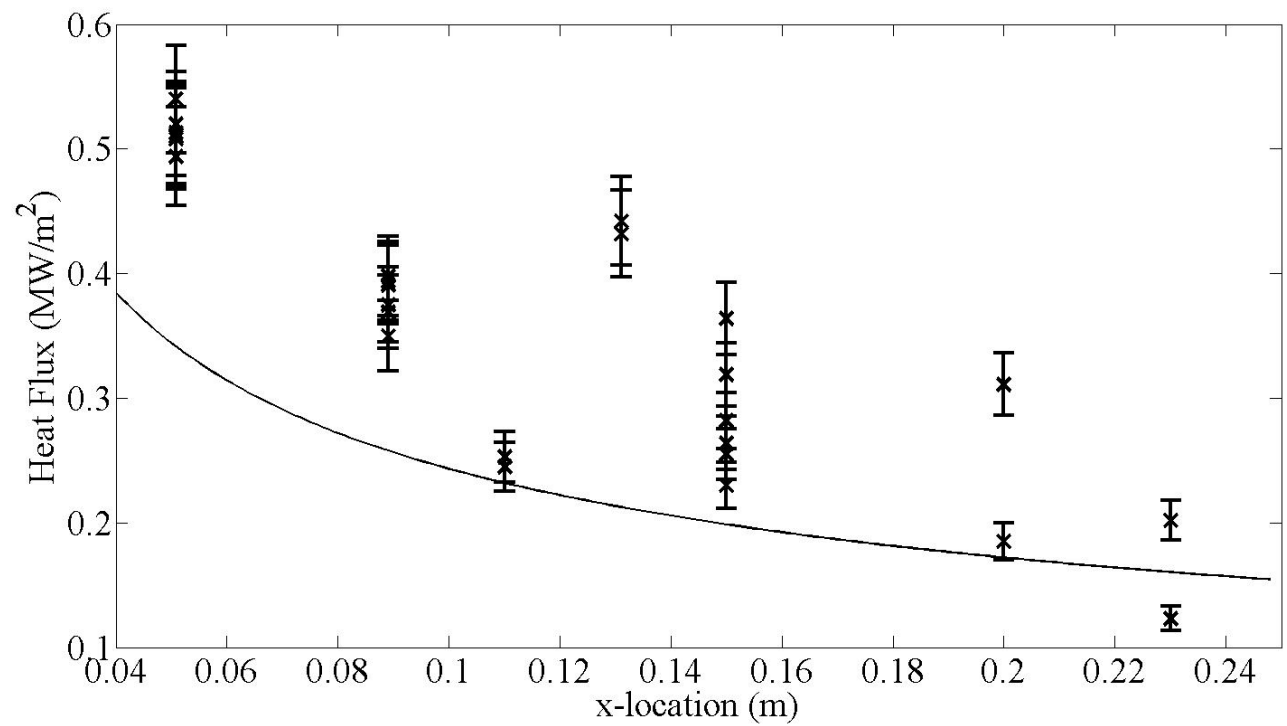

Figure 4: Experimental heat flux measurements over the flat plate data model $(\times)$ and comparison with theoretical prediction (-). ${ }^{22}$

\section{Results}

\section{IV.A. Flat plate results}

Data were first taken for the flat plate model. This model geometry was chosen due to both its simple geometry and the existence of analytical equations to predict the heat flux. The establishment time of a steady boundary layer was taken into account when analyzing these data using the work of Gupta, ${ }^{23}$ who performed an analysis on the time required for the boundary layer to relax to steadiness on a flat plate in an expansion tube. Two different modes of expansion tube operation were identified, the Mirels limit and the Blasius limit. In the Mirels limit, the transmitted shock from the rupture of the secondary diaphragm and the contact surface are so close together that the time between their arrivals goes to zero. In the Blasius limit the transmitted shock and the contact surface are sufficiently far apart that the time between them can be said to go to infinity. For the run condition used in this study it is reasonable to assume we are in the Blasius limit. In this regime, a Blasius boundary layer is established over the flat plate during the time in which the accelerator gas is passing over it. With the arrival of the contact surface, this boundary layer is washed away, and a new boundary layer consisting of test gas must be formed. During this transition, there is a finite amount of time when the boundary layer will consist of both accelerator and test gas while it is simultaneously relaxing to a steady state. Gupta performed a series of numerical calculations and determined that there was a critical value for the non-dimensional parameter $\alpha$ after which steady boundary layer flow would be established in the test gas. The non-dimensional parameter is defined as:

$$
\alpha=\frac{L}{u_{e} t}
$$

where $t$ is the time coordinate beginning with the arrival of the contact surface, $u_{e}$ is the freestream velocity and $L$ is the distance along the plate. Gupta determined that with $\alpha=0.3$ the boundary layer would have relaxed to a state where it is completely composed of test gas and is steady. Using the theoretically calculated freestream velocity, it is possible to compute the time to reach steadiness at each thermocouple location along the plate. Theoretical predictions for the laminar heat transfer were calculated with the reference enthalpy method of Simeonides. ${ }^{22}$ Figure 4 shows the results of these tests.

\section{IV.B. Curved and linear ramp results}

The heat flux distribution over the Curved25 model is shown in Figure 5. The comparison of the Curved25 versus the flat plate data can be seen in Figure 6. Significant increases in heat transfer with curvature over 


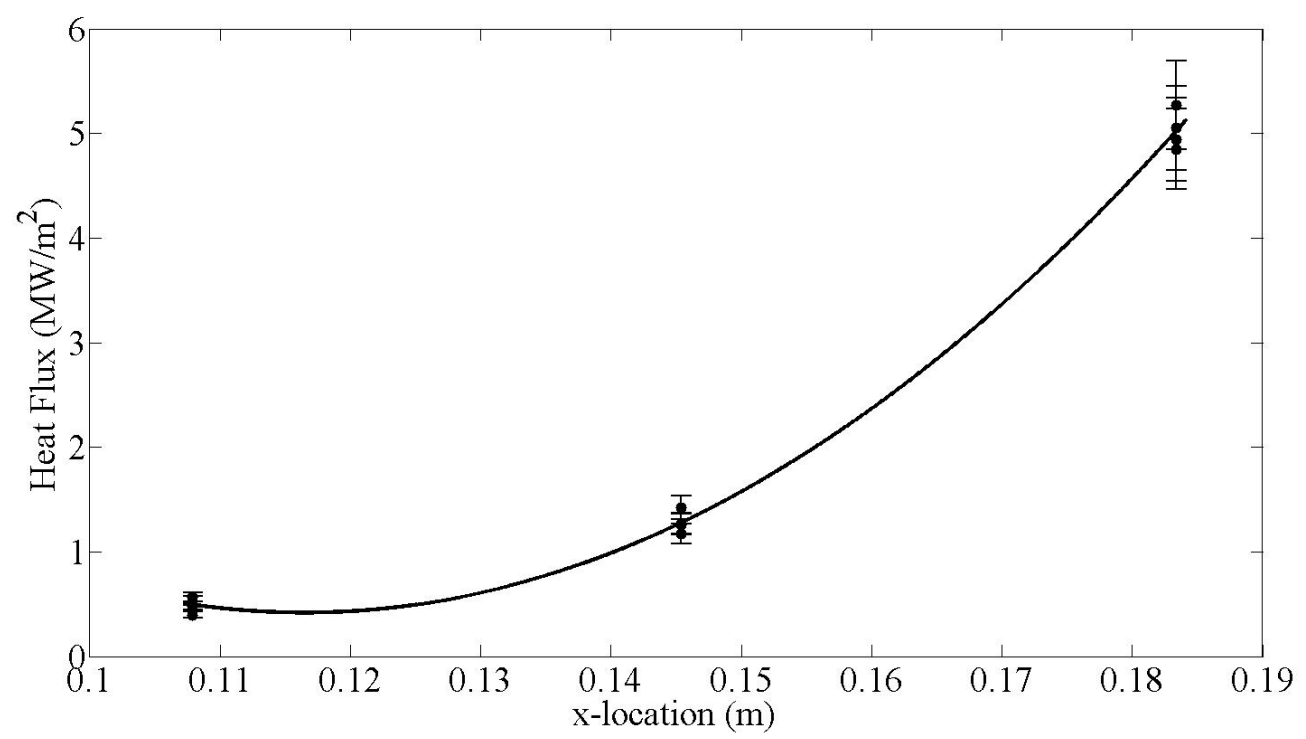

Figure 5: Heat flux distribution over curved surface (Curved25 model).

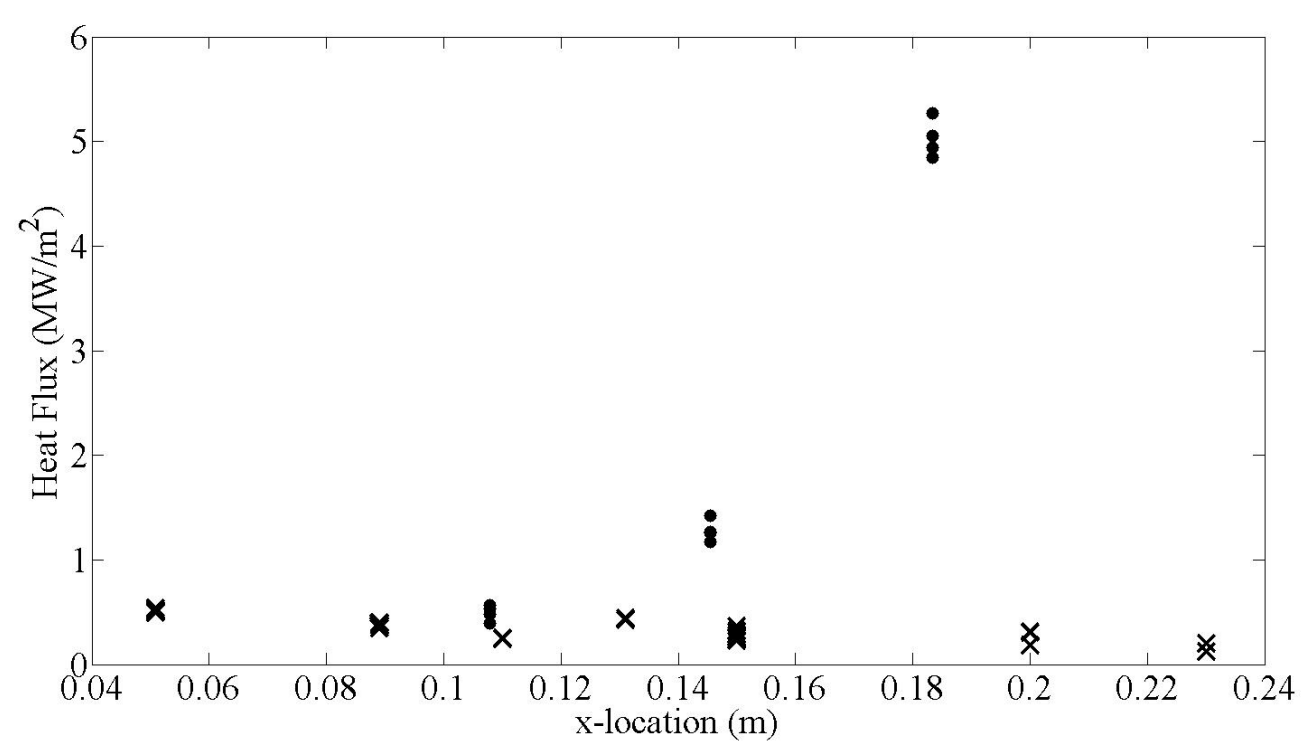

Figure 6: Comparison of flat plate $(\times)$ and Curved25 $(\bullet)$ heat flux.

the flat plate results can clearly be seen. With increasing streamwise distance, the percent increase between the flat plate and curved ramp values increases. Values are tabulated in Table 3.

The heat flux data taken along the centerline of the Curved16 model is presented in Figure 7 . The three segments of the model (flat plate, curved, and linear) are identified in the figure. As expected, similar heating levels are seen at the first two points on Curved25 and the corresponding points on Curved16. Similar to the data from Curved25, a significant increase in heat transfer in the x-direction is observed. One interesting feature of the data over Curved16 is the discrepancy between the centerline and off-centerline heat transfer data. The centerline and off-centerline data over Curved16 are shown in Figure 8. Initially, it was thought that this could be due to flow spillage due to the aspect ratio of the model. The fact that this behavior was not observed on the flat plate and Curved25 models is consistent since on these models thermocouples were only located on the centerline.

In order to investigate the effects of aspect ratio, attachments were made for the flat plate model which would double its width. In addition, four thermocouples were added at the third thermocouple position at selected span wise locations. These same thermocouples were used both with and without the side 




Figure 7: Centerline heat flux data over the Curved16 model.

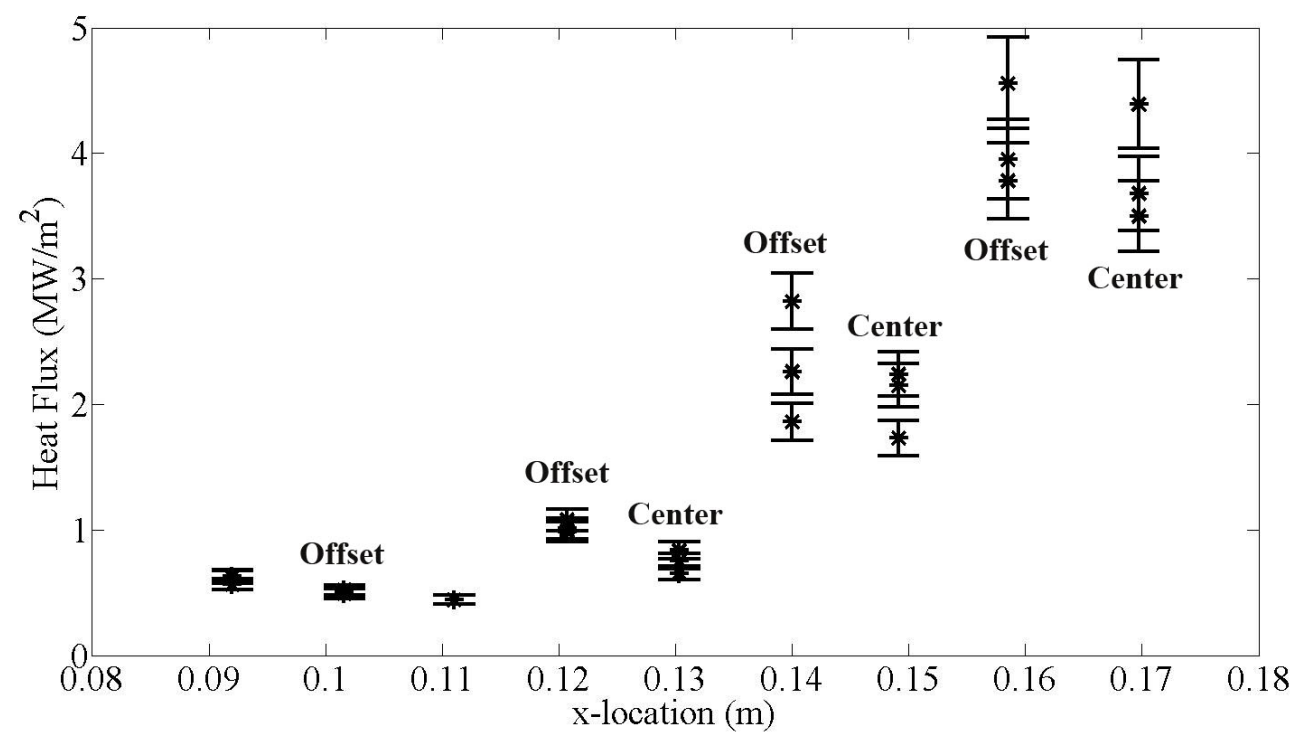

Figure 8: Comparison of centerline and off-centerline heat flux data over the Curved16 model.

attachments to provide the best indicator of any three dimensional effects due to flow spillage. Figure 9 shows the data collected both with and without the side attachments. The discrepancy in the heat transfer data at the very edges of the plate indicates there is a significant effect due to the width of the plate. Closer to the centerline, however, there seems to be little to no difference in the heat transfer between the wide and thin flat plates. This indicates that there is some flow spillage around the sides of the model, but that close to the centerline this does not affect the heat transfer. Since the thermocouples on Curved16 are all located close to the centerline (between the distance between the centerline and first span wise thermocouple in the span wise tests) it is reasonable to say that flow spillage is not causing the "stepped" behavior seen in the data over Curved16. A possible explanation for this anomaly is that streamwise oriented vortices are forming over the model causing a fluctuation in heat transfer. It has been found in previous studies ${ }^{14}$ that the formation of Görtler vortices can result in fluctuation in heat flux of up to $30 \%$. The radius of curvature of this ramp is such that it should promote the formation of Görtler vortices. More investigation is needed to determine if vortices are forming over the model, and if they are responsible for these deviations in heat flux. 


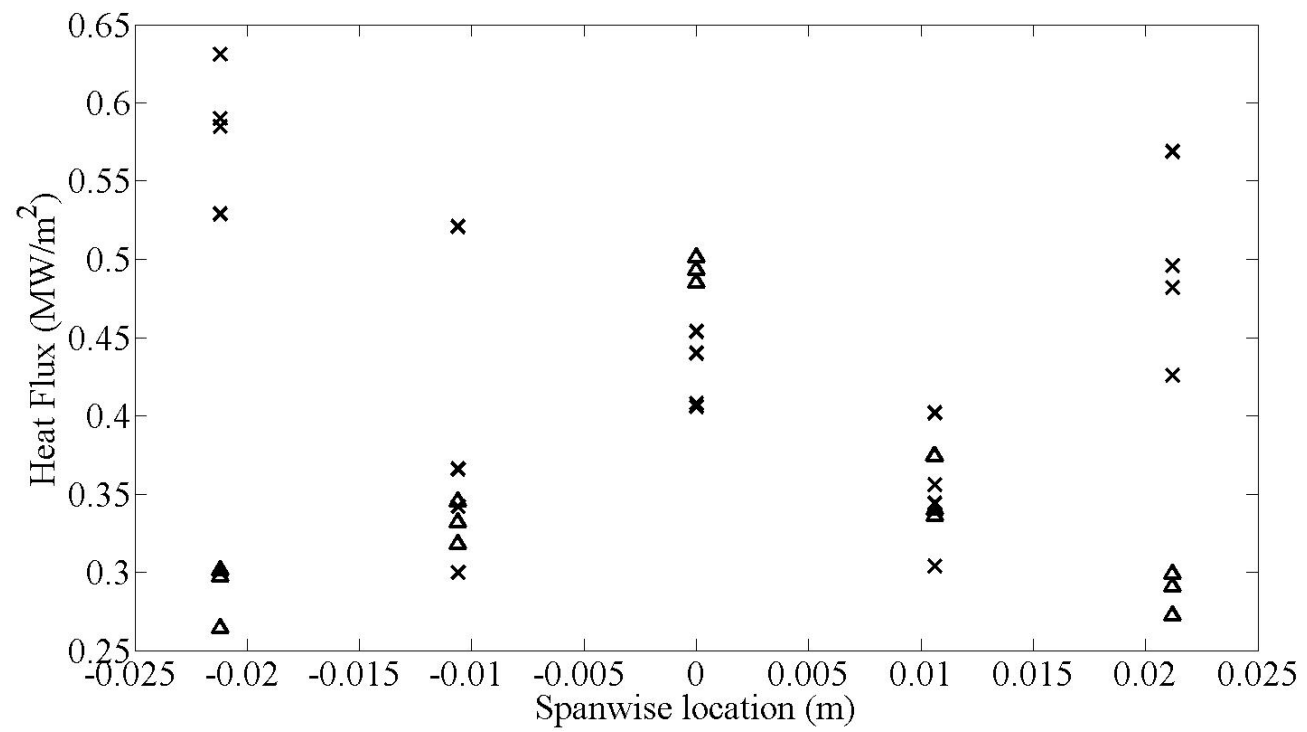

Figure 9: Spanwise heat flux distributions over flat plate models with $(\times)$ and without $(\triangle)$ side attachments extending the width of the plate.

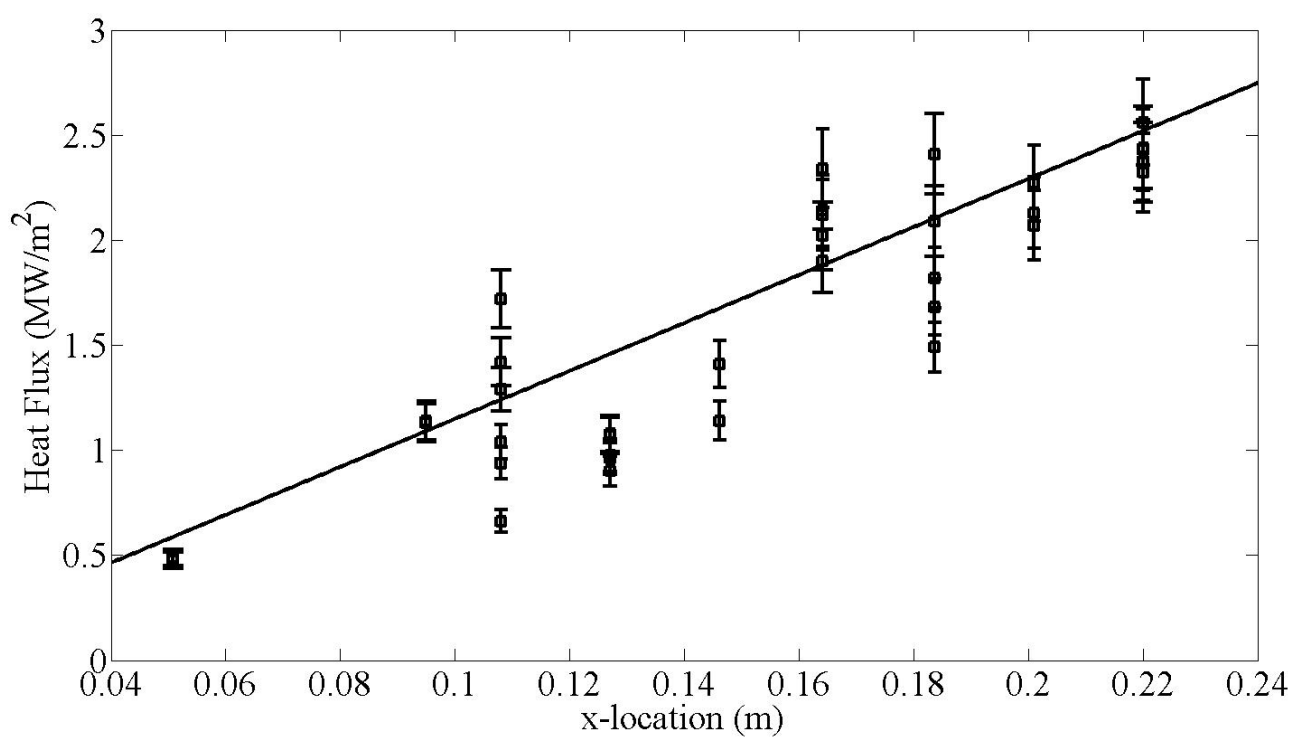

Figure 10: Heat flux data over the Linear10 model.

The heat flux distribution over the Linear10 model is show in Figure 10. Here again a trend of increasing heat flux in the $\mathrm{x}$-direction is observed. The heat flux increase is similar to the two curved ramp models before approximately 0.14 meters, but after that appears to increase at a slower rate. The Linear10 data does not exhibit the same "stepped" behavior as the Curved16 data. This is important since the Linear10 model also has the same off-centerline thermocouple design as Curved16. The conclusion that stepped behavior is not due to any flow spillage effects over the model was further supported by recent experiments on these same models using a novel fast pressure sensitive paint (PSP) developed by Innovative Scientific Solutions Inc. Tests over the same model coated with the PSP showed little to no spanwise variation in pressure, indicating the three dimensional effects are not significant over these models. ${ }^{24}$

Curve fits to the curved ramp models were made. The least squares fit for Curved 25 model with increasing streamwise distance $x$, shown in Figure 5 , is

$$
q=1000 x^{2}-240 x+11
$$


Table 3: Increase in ratio of curved ramp (Curved25) over flat plate heat transfer with distance $x$ from the leading edge.

\begin{tabular}{|c|c|c|}
\hline$x=0.12 m$ & $x=0.15 m$ & $x=0.18 m$ \\
\hline 2.11 & 2.79 & 7.32 \\
\hline
\end{tabular}

where $q$ is the heat flux, while for the Curved16 model, Figure 7, the fit is

$$
q=940 x^{2}-200 x+14
$$

As shown, fits to the Curved25 and Curved16 data are best described by a quadratic function, whereas a fit to the Linear10 data, Figure 10, is best described as linear, and is given by

$$
q=11 x+0.88
$$

Since the surface equations of Curved25 and Curved16 are quadratic, and the surface equation of Linear10 is obviously linear this suggests that the rate of heat flux increase is directly linked to the equation of the surface of the model. Further testing with a model with a cubic surface equation would help to expand on this result.

\section{Conclusions}

This study has examined the effect of wall curvature on heat transfer in a hypersonic flow. Four model geometries were investigated: a flat plate, a linear ramp, a curved ramp with 25 degree turning angle (denoted Curved25), and a ramp with a 16 degree turning angle section (denoted Curved16) which was selected to match the experiments of Donovan et al ${ }^{10}$ Baseline measurements over the flat plate model showed experimental heat transfer distributions were in reasonable agreement with theoretical prediction. Measurements over the two models with concave surface curvature showed significant increase in heat flux with increasing streamwise distance over flat plate values. Over the Curved25 model, the heat transfer increased by a factor of seven. This increase is due to compound effects of the surface curvature as well as the adverse pressure gradient. For comparative purposes, heat transfer measurements were made over a compression ramp with no curvature. It was found that the increase in heat transfer along a model is linked to the geometry of the surface. Over the curved models with surface best described by a quadratic the fit to the experimental heat transfer was quadratic. For the linear ramp, the best fit to the experimental heat flux data was linear.

\section{Acknowledgments}

This work was funded through the Air Force Office of Scientific Research FA9550-08-1-0172 with Dr. John Schmisseur as program manager. We are grateful to the Caltech T5 group, Prof. Hans Hornung, Bahram Valiferdowski, Drs Eric Marineau, Adam Rasheed, and Ivett Leyva for their help with thermocouples. The authors would like to thank Ryan Fontaine, Manu Sharma, and Andy Swantek for their help with this work.

\section{References}

\footnotetext{
${ }^{1}$ Malo-Molina, F. J., Gaitonde, D. V., and Kutschenreuter, P. H., "Numerical Investigation of an Innovative Inward Turning Inlet," 17th Computational Fluid Dynamics Conference, AIAA, Toronto, Ontario, Canada, June 2005.

${ }^{2}$ Malo-Molina, F. J., Gaitonde, D. V., Ebrahimi, H., and Ruffin, S. M., "High Fidelity Flowpath Analysis of a Supersonic Combustor Coupled to Innovative Inward-Turning Inlets," 17th Aerospace Sciences Meeting, AIAA, Orlando, FL, January 2009.

${ }^{3}$ Gaitonde, D. V., Malo-Molina, F. J., Croker, B., and Ebrahimi, H., "The Flowfield Structure in Generic Inward-TurningBased Propulsion Flowpath Components," 43rd Joint Propulsion Conference, AIAA, Cincinnati, OH, July 2007.

${ }^{4}$ Drayna, T. W., Nompelis, I., and Candler, G. V., "Hypersonic Inward Turning Inlets: Design and Optimization," 44th Aerospace Sciences Meeting, AIAA, Reno, NV, Jan 2006.

${ }^{5}$ Walker, S., Rodgers, F., and Esposita, A., "Hypersonic collaborative Australia/United States Experiment (HYCAUSE)," AIAA/CIRA 13th Int. Space Planes and Hypersonics Systems and Technologies, AIAA, 2005.

${ }^{6}$ Walker, S. and Rodgers, F., "Falcon hypersonic technology overview," AIAA/CIRA 13th Int. Space Planes and Hypersonics Systems and Technologies, AIAA, 2005.
} 
${ }^{7}$ Billig, F., Baurle, R., Tam, C., and Wornom, S., "Design and Analysis of Streamline Traced Hypersonic Inlets," 9th International Space Planes and Hypersonic Systems and Technologies Conference and 3rd Weakly Ionized Gases Workshop, AIAA, Norfolk, VA, November 1999.

${ }^{8}$ Molder, S. and Szpiro, E., "Busemann inlet for hypersonic speeds," Journal of Spacecraft and Rockets, Vol. 3, No. 8, 1966, pp. 1172-1176.

${ }^{9}$ Billig, F. and Kothari, A., "Streamline tracing: Technique for designing hypersonic vehicles," Journal of Propulsion and Power, Vol. 16, No. 3, 2000, pp. 465-471.

${ }^{10}$ Donovan, J., Spina, E., and Smits, A., "The Structure of a Supersonic Boundary Layer Subject to Concave Wall Curvature," Journal of Fluid Mechanics, Vol. 259, January 1994, pp. 1-24.

${ }^{11}$ Ekoto, I. W., Bowersox, R. D., Beutner, T., and Goss, L., "Response of supersonic turbulent boundary layers to local and global mechanical distortions," Journal of Fluid Mechanics, Vol. 630, 2009, pp. 225-265.

${ }^{12}$ Holden, M. and Chadwick, K., "Studies of Laminar, Transitional and Turbulent Hypersonic Flows Over Curved Compression Surfaces," 33rd Aerospace Sciences Meeting and Exhibit, AIAA, January 1995.

${ }^{13}$ Ciolkosz, L. and Spina, E., "An Experimental Study of Goertler Vortices in Compressible Flow," Experiments in Fluids, Vol. 16, 1993, pp. 10-16.

${ }^{14}$ de Luca, L., Cardone, G., de la Chevalerie, D., and Fonteneau, A., "Goertler Instability of a Hypersonic Boundary Layer," Experiments in Fluids, Vol. 16, 1993, pp. 10-16.

${ }^{15}$ Dufrene, A., Sharma, M., and Austin, J. M., "Design and Charachterization of a Hypervelocity Expansion Tube Facility," Journal of Propulsion and Power, Vol. 23, No. 6, Nov 2007, pp. 1185-1193.

${ }^{16}$ Sanderson, S. R., Shock wave interaction in hypervelocity flow, Ph.D. thesis, California Institute of Technology, Pasadena, California, 1995.

${ }^{17}$ Davis, J.-P., High-Enthalpy Shock/Boundary-Layer Interaction on a Double Wedge, Ph.D. thesis, California Institute of Technology, Pasadena, California, 1999.

${ }^{18}$ Rasheed, A., Passive Hypervelocity Boundary Layer Control Using an Ultrasonically Absorptive Surface, Ph.D. thesis, California Institute of Technology, Pasadena, California, 2001.

${ }^{19}$ Croarkin, M. C., Guthrie, W. F., Burns, G. E., Kaeser, M., and Strouse, G. F., "Temperature-Electromotive Force Referance Function and Tables for the Letter-Designated Thermocouple Types Based on the ITS-90." Monograph 175, National Institute of Standard Technologies, 1993.

${ }^{20}$ Flaherty, W. and Austin, J. M., "Comparative surface heat transfer measurements in hypervelocity flow," 48th AIAA Aerospace Sciences Meeting, AIAA, Jan 2010.

${ }^{21}$ McGilvray, M., Austin, J., Sharma, M., Jacobs, P., and Morgan, R., "Diagnostic Modelling of an Expansion Tube Operating Condition," Shock Waves, Vol. 19, No. 1, 2009, pp. 59-66.

${ }^{22}$ Simeonides, G., "Gerneralized reference enthalpy formulations and simulation of viscous effects in hypersonic flows," Shock Waves, Vol. 8, 1998, pp. 161-172.

${ }^{23}$ Gupta, R. N., "An Analysis of the Relaxation of Laminar Boundary Layer on a Flat Plate After Passage of an Interface with Application to Expansion-Tube Flows," Tech. Rep. TR R-397, NASA, 1973.

${ }^{24}$ Flaherty, W., Crafton, J., Elliott, G. S., and Austin, J. M., "Application of fast pressure sensitive paint in hypervelocity flow," 49th Aerospace Sciences Meeting (pending acceptance), AIAA, Orlando, FL, Jan 2011. 